\title{
Retrospective evaluation of the effectiveness of indoor residual spray with pirimiphos-methyl (Actellic) on malaria transmission in Zambia
}

Joseph Keating ${ }^{1 *} \mathbb{D}$, Joshua O. Yukich ${ }^{1}$, John M. Miller ${ }^{2}$, Sara Scates ${ }^{1}$, Busiku Hamainza ${ }^{3}$, Thomas P. Eisele ${ }^{1}$ and Adam Bennett ${ }^{4}$

\begin{abstract}
Background: Widespread insecticide resistance to pyrethroids could thwart progress towards elimination. Recently, the World Health Organization has encouraged the use of non-pyrethroid insecticides to reduce the spread of insecticide resistance. An electronic tool for implementing and tracking coverage of IRS campaigns has recently been tested (mSpray), using satellite imagery to improve the accuracy and efficiency of the enumeration process. The purpose of this paper is to retrospectively analyse cross-sectional observational data to provide evidence of the epidemiological effectiveness of having introduced Actellic 300CS and the mSpray platform into IRS programmes across Zambia.
\end{abstract}

Methods: Health facility catchment areas in 40 high burden districts in 5 selected provinces were initially targeted for spraying. The mSpray platform was used in 7 districts in Luapula Province. An observational study design was used to assess the relationship between IRS exposure and confirmed malaria case incidence. A random effects Poisson model was used to quantify the effect of IRS (with and without use of the mSpray platform) on confirmed malaria case incidence over the period 2013-2017; analysis was restricted to the 4 provinces where IRS was conducted in each year 2014-2016.

Results: IRS was conducted in 283 health facility catchment areas from 2014 to 2016; 198 health facilities from the same provinces, that received no IRS during this period, served as a comparison. IRS appears to be associated with reduced confirmed malaria incidence; the incidence rate ratio (IRR) was lower in areas with IRS but without mSpray, compared to areas with no IRS (IRR $=0.91,95 \% \mathrm{Cl} 0.84-0.98$ ). Receiving IRS with mSpray significantly lowered confirmed case incidence (IRR $=0.75,95 \% \mathrm{Cl} 0.66-0.86)$ compared to no IRS. IRS with mSpray resulted in lower incidence compared to IRS without mSpray (IRR $=0.83,95 \% \mathrm{Cl} 0.72-0.95)$.

Conclusions: IRS using Actellic-CS appears to substantially reduce malaria incidence in Zambia. The use of the mSpray tool appears to improve the effectiveness of the IRS programme, possibly through improved population level coverage. The results of this study lend credence to the anecdotal evidence of the effectiveness of 3GIRS using Actellic, and the importance of exploring new platforms for improving effective population coverage of areas targeted for spraying.

Keywords: Pirimiphos-Methyl (Actellic), Malaria, Zambia, Indoor Residual Spray, mSpray

${ }^{*}$ Correspondence: jkeating@tulane.edu

${ }^{1}$ Center for Applied Malaria Research and Evaluation, Department of Tropical Medicine, School of Public Health and Tropical Medicine, Tulane University, 1440 Canal Street, Suite 2320, New Orleans 70112, USA

Full list of author information is available at the end of the article

\section{Background}

Vector control through the use of long-lasting insecticidal nets (LLINs), and the use of indoor residual spraying (IRS), have been a cornerstone to recent malaria

c) The Author(s) 2021. This article is licensed under a Creative Commons Attribution 4.0 International License, which permits use, sharing, adaptation, distribution and reproduction in any medium or format, as long as you give appropriate credit to the original author(s) and the source, provide a link to the Creative Commons licence, and indicate if changes were made. The images or other third party material in this article are included in the article's Creative Commons licence, unless indicated otherwise in a credit line to the material. If material is not included in the article's Creative Commons licence and your intended use is not permitted by statutory regulation or exceeds the permitted use, you will need to obtain permission directly from the copyright holder. To view a copy of this licence, visit http://creativecommons.org/licenses/by/4.0/. The Creative Commons Public Domain Dedication waiver (http://creativecommons.org/publicdomain/zero/1.0/) applies to the data made available in this article, unless otherwise stated in a credit line to the data. 
control success $[1,2]$.

However, widespread insecticide resistance to pyrethroids is a major threat to continued progress towards elimination [3]. Recently, the Global Plan for Insecticide Resistance Management (GPIRM) of the World Health Organization (WHO) has encouraged the use of nonpyrethroid insecticides as a part of GPIRM best practices [4] to reduce the spread of insecticide resistance.

Programs considering new or additional insecticides for IRS lack rigorous field-based evidence on the effectiveness of next (3rd) generation pyrethroid-free insecticides for IRS (3GIRS). In addition, information on the combination of vector control tools is needed, given the high cost of program rollout. As the effectiveness of pyrethroid-based insecticides is well documented for both LLINs and IRS [1, 2, 5], and IRS is considered a costeffective vector control intervention in many settings [6], a logical next step would be to assess whether adding new 3GIRS chemicals improves malaria prevention and control practices.

The WHO recommends a threshold of $85 \%$ community-level coverage of IRS to maintain effectiveness. A study conducted on Bioko Island demonstrated that if the community is covered at or above an $80 \%$ threshold, houses not receiving IRS see the same benefits as their sprayed neighbors through a community effect [7]. Therefore, effective spatial coverage of IRS is critical; the location of all structures in the program area must be enumerated or mapped to increase the likelihood that the $85 \%$ threshold is reached. Until recently, this was done by teams of enumerators, a time consuming and costly process that often led to a number of structures being missed. While this is better than not enumerating, this can result in high 'reported operational coverage' (i.e. the reported number of structures sprayed out of the total structures enumerated), but poor 'effective population coverage' (i.e. the number of structures sprayed out of the total eligible structures actually present within an area).

A new electronic tool for implementing and tracking coverage of IRS campaigns in Zambia has recently been tested. This tool, mSpray (now called REVEAL), is a mobile and desktop-based platform that utilizes satellite imagery to improve the accuracy and efficiency of the enumeration process by ensuring all eligible structures are identified [8]. While mSpray technology should, theoretically, increase 'effective population coverage' and thus the effectiveness of IRS due to the improved communityeffect of the intervention, it may yield lower 'reported operational coverage' due to the identification of additional structures that increase the target-area denominator. By recording and tracking spray operation data at the structure level, a more thorough accounting of both sprayable and non-sprayable structures is achieved.
The Zambian Government has identified malaria control as a priority, with the vision of a malaria elimination [9]. Since the reintroduction of IRS in Zambia in 2000, IRS has been a cornerstone of malaria vector control in the country, effectively scaling up from 5 districts in 2003 to all districts (72 in 2011, now 117 as of 2018) by 2011 [10]. From 2000 to 2009, vector control relied exclusively on DDT and pyrethroids, both of which share the same mode of action. In 2009, resistance to both insecticide classes was detected, prompting the formation of the Insecticide Resistance Management Technical Working Group (IRMTWG) in 2010 [11, 12]. In 2011, the use of DDT was discontinued, and carbamates and organophosphates were introduced. Due to growing concerns of documented resistance in all areas of Zambia to all classes of insecticides, except the organophosphates, Actellic 300CS, a microencapsulated formulation of the organophosphate insecticide pirimiphos-methyl, was introduced in 2013 [10]. While Actellic 300CS is thought to be more effective than pyrethroid-based chemicals because of insecticide susceptibility, it is considerably more expensive [13-15]. A study in Migori County, Kenya recently demonstrated that a single application of IRS with Actellic 300CS provided ten months protection, resulted in the near elimination of the primary malaria vector Anopheles funestus, and reduced malaria case counts among febrile outpatients [12]. However, empirical evidence of the effectiveness of Actellic 300CS on human health outcomes is quite limited and programmes and donors are increasingly looking for rigorous evidence of effectiveness when considering new interventions [16].

The use of LLINs, IRS and investigational vector control tools such as 3GIRS with Actellic 300CS in Zambia provides an opportunity for a retrospective evaluation of the effectiveness of 3GIRS across different scenarios. The purpose of this paper is to retrospectively analyse crosssectional observational data to provide evidence of the epidemiological effectiveness of having introduced Actellic 300CS into IRS programmes across Zambia as well as the effectiveness of the mSpray platform for increasing spray coverage.

\section{Methods}

\section{Study setting}

This study was conducted in Luapula, Northern, Muchinga, and Eastern provinces in Zambia. Malaria transmission in these provinces peaks from December to June following the seasonal rains. The Zambia National Malaria Elimination Centre (NMEC) has successfully scaled up the main WHO-recommended malaria control interventions (i.e. LLINs, IRS, and improved access to prompt effective treatment of malaria) over the past decade and is now considering alternative strategies to 
further reduce the malaria burden [17]. Table 1 lists study vector control coverage and parasite prevalence by study province [18].

Mass ITN campaigns are conducted periodically throughout the country, with campaigns occurring in 2014 and late 2017. IRS campaigns are conducted annually, ideally from October to November, just prior to the onset of the seasonal rains. The 2018 MIS reported $80 \%$ of Zambian households owning at least one ITN, up from $62 \%$ to 2008 ; and $34.8 \%$ of Zambian households having been sprayed with insecticide in the previous 12 months up from $14 \%$ to 2008 [19].

\section{PMI Africa indoor residual spraying project (AIRS) and $\mathrm{mSpray}$ implementation}

In 2014, AIRS Zambia identified 40 high burden districts in 5 selected provinces, and subdistrict catchment areas were targeted for spraying with pirimiphos-methyl [20]. Criteria for inclusion included areas where recent national prevalence surveys suggest high burden, operational areas where PMI operates and programmatic information on insecticide resistance patterns [21]. The mSpray platform was used in 7 districts in Luapula Province. The mSpray platform is a tool used to improve IRS enumeration, targeting, and spraying. It is a cloud-based data recording and management system that allows spray personnel to collect spray data and GPS coordinates electronically in the field using a tablet. Data are submitted to the cloud for immediate viewing of spray campaign progress in relation to satellite-based and field-verified structure enumeration data. Spraying began in 2014 and was repeated in 2015 and again in 2016. Spraying was only conducted in Central Province in 2014 and 2015, and in a smaller number of catchment areas, and was excluded from the analysis.

\section{Study design}

An observational study design was used to assess the relationship between IRS exposure and confirmed malaria case incidence. The primary outcome was confirmed outpatient (OPD) malaria case incidence among all ages during the high transmission season (Dec-June) between 2013 and 2017: defined as the number of OPD confirmed malaria cases at the health facility catchment

Table 1 Study setting LLIN, IRS and under 5-year-old malaria prevalence status in 2015

\begin{tabular}{lllll}
\hline Province & Luapula & Northern & Muchinga & Eastern \\
\hline \%HH LLIN ownership & 88.6 & 78.9 & 82.2 & 94.5 \\
\% HH sprayed with IRS & 31.6 & 17.5 & 27.7 & 56.0 \\
Malaria prevalence $(<5)$ & 32.5 & 27.6 & 21.4 & 12.7 \\
\hline
\end{tabular}

level, standardized per catchment population, as reported to the health management information system (HMIS). Case confirmations included both malaria RDT and microscopy reporting. Exposure was defined at the health facility catchment level by having IRS activities conducted in the preceding season.

\section{Data sources}

Data on IRS coverage were collected from the AIRS and mSpray programmes: defined as the total number of households sprayed by date within target areas. Monthly enhanced vegetation indices (EVI) from MODIS satellite imagery (https://lpdaac.usgs.gov/products/mod13 a3v006/) and monthly rainfall data from the CHIRPs dataset (https://www.nature.com/articles/sdata201566) were also extracted at the health facility level for those facilities with available geographical coordinates as control variables in the analysis.

\section{Data analysis}

All health facility catchment areas within the study area were categorized as either having received IRS using the mSpray platform, receiving IRS without mSpray, or receiving no IRS, for the spray seasons 2014, 2015, and 2016. For descriptive comparisons across all catchment areas, raw operational coverage estimates were calculated by dividing the numbers of structures reported sprayed by the programme, by the numbers of structures reported as found. As the numbers of structures found were sometimes calculated differently for mSpray and non-mSpray areas, the total number of structures sprayed per population for each health facility catchment was also compared; this value was calculated as the total number of reported structures sprayed divided by the catchment area population as estimated from administrative records.

Catchment area health facilities were matched to the number of confirmed malaria cases per month over 2013-2017, the number of tests conducted per month over 2013-2017, and the estimated catchment population size. The total number of confirmed malaria cases was calculated over the high transmission season (December-June, following each IRS spray campaign) for each facility. Facilities that had poor reporting (data for fewer than 3 of the 7 high transmission months available) were removed from the analysis. December-June incidence rates per 1000 were calculated by dividing the total December-June confirmed cases by the catchment population and multiplying by 1000 . Total rainfall data were extracted for facilities with geographical coordinates and lagged by two months (November-April), and the mean enhanced vegetation index (EVI) was calculated for each facility and lagged by one month (December-May). 
A random effects Poisson model was used to quantify the effect of IRS (with and without use of the mSpray platform) on January-June confirmed case incidence at the catchment level over the period 2013-2017. The primary effect was estimated using a categorical dummy variable representing "no IRS", "IRS", or "IRS with mSpray" for each health catchment area. Province and year fixed effects were included to control for regional and temporal trends. The total number of tests conducted controlled for variable testing rates, and the previous year's total confirmed cases were included to control for temporal autocorrelation. Rainfall and EVI were included as covariates to control for the overall propensity of the area to harbour mosquito populations.

\section{Results}

IRS was conducted in a total of 283 catchment areas from 2014 to 2016 and expanded over time: 219 in 2014, 243 in 2015, and 255 in 2016. mSpray was used at

Table 2 Number of health facilities included in the analysis by year and IRS status

\begin{tabular}{lrrrrr}
\hline Category & 2013 & 2014 & 2015 & 2016 & 2017 \\
\hline Included in descriptive analysis & & & & \\
No IRS & 481 & 481 & 262 & 238 & 226 \\
IRS & 0 & 0 & 168 & 191 & 202 \\
IRS W/ mSpray & 0 & 0 & 51 & 52 & 53 \\
Included in regression & & & & & \\
No IRS & 392 & 392 & 207 & 189 & 180 \\
IRS & 0 & 0 & 137 & 153 & 164 \\
IRS W/ mSpray & 0 & 0 & 48 & 50 & 48 \\
\hline
\end{tabular}

66 health facility catchment areas in Luapula province from 2014 to 2016: 51 received mSpray in 2014, 52 in 2015, and 53 in 2016. After linking with geographical and environmental data, a total of 185 IRS catchment areas (48 mSpray) were available for the regression analysis in 2014; 203 (50 mSpray) in 2015; and 212 (48 mSpray) in 2016 (Table 2). A total of 157 health facilities that received no IRS during this period were available for comparison in the regression analysis (Fig. 1).

Confirmed malaria case incidence per 1000 rose slightly over the period of observation 2013-2017. Confirmed case incidence by year leveled out in those receiving IRS and decreased in 2016 and 2017 in those receiving IRS with mSpray (Fig. 2). Reported operational coverage estimates were high for both IRS and IRS/mSpray, but slightly higher for IRS without mSpray (Table 3). Conversely, the number of structures sprayed per population was slightly higher for IRS with mSpray across all years (Table 3 ).

Receiving IRS appears to be associated with reduced confirmed malaria incidence; the incidence rate ratio (IRR) was lower in areas with IRS but without mSpray, compared to areas with no IRS, but the effect size was smaller (IRR $=0.91,95 \%$ CI 0.84-0.98) (Table 4). Receiving IRS with mSpray was significantly protective against confirmed case incidence (IRR $=0.75,95 \% \mathrm{CI}$ 0.66-0.86) compared to no IRS. Compared directly, IRS with $\mathrm{mSpray}$ resulted in lower incidence than IRS without $\mathrm{mSpray}$ (IRR $=0.83,95 \%$ CI $0.72-0.95)$.

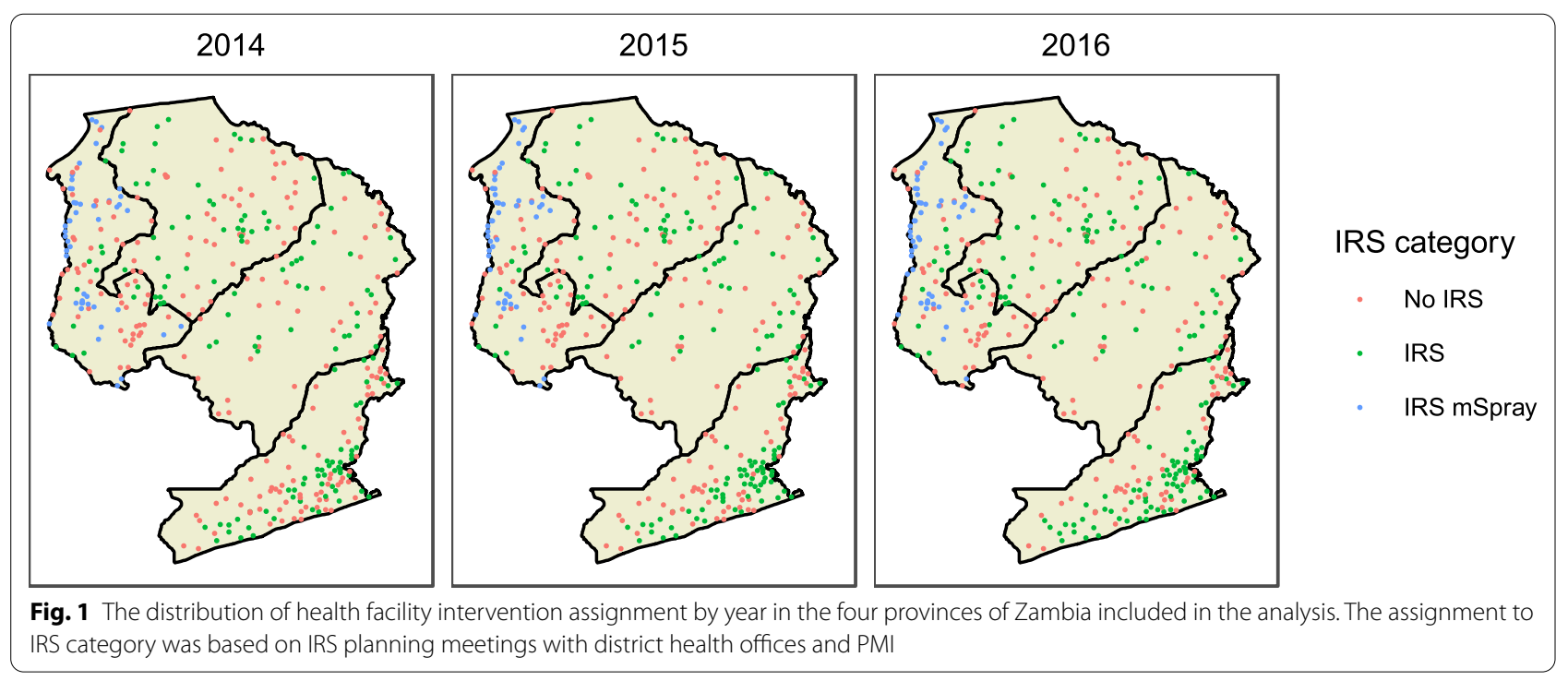



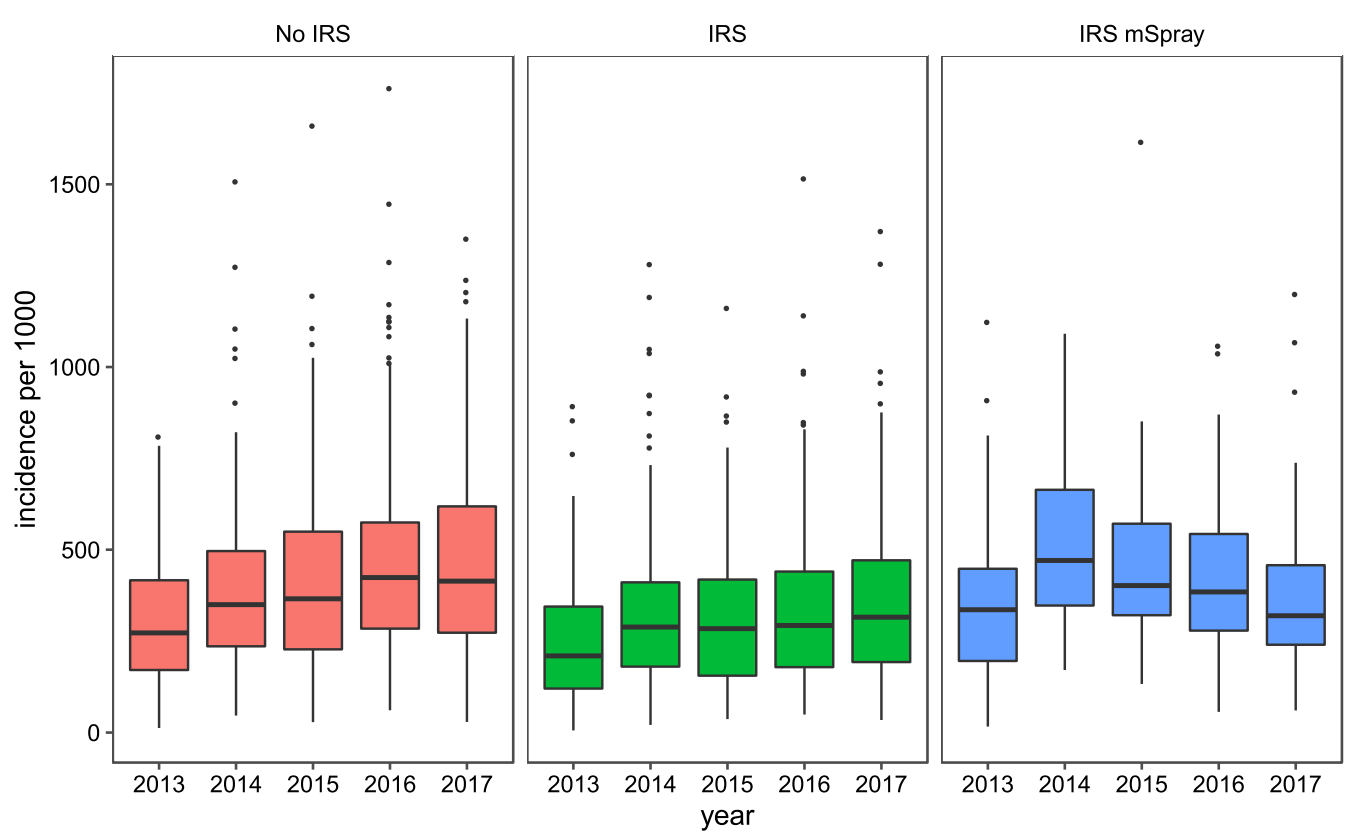

Fig. 2 Confirmed malaria case incidence per 1000 (Dec-Jun) by year for facilities with a no IRS, b IRS, and c IRS with mSpray

Table 3 Coverage estimates and confidence intervals by IRS type

\begin{tabular}{llll}
\hline Coverage estimate & 2014 & 2015 & 2016 \\
\hline $\begin{array}{l}\text { Operational coverage } \\
\text { IRS }\end{array}$ & $94.7(93.6-95.9)$ & $94.3(93.2-95.4)$ & $92.4(91.4-93.4)$ \\
IRS with mSpray & $87.0(83.5-90.5)$ & $95.0(93.8-96.3)$ & $88.8(87.4-90.1)$ \\
Structures per population & & \\
IRS & $0.13(0.11-0.14)$ & $0.17(0.15-0.18)$ & $0.17(0.15-0.20)$ \\
IRS with mSpray & $0.14(0.11-0.17)$ & $0.19(0.14-0.23)$ & $0.21(0.16-0.25)$ \\
\hline
\end{tabular}

\section{Discussion}

This analysis used multiple datasets linked spatially and temporally to assess the relationship between IRS using Actellic-CS, IRS using the mSpray platform, no IRS, and malaria incidence at a subnational level in Zambia. Total confirmed malaria case incidence has increased slightly over this period as diagnostic capacity has also increased [19]. However, this analysis showed a consistent association between implementation of IRS and lower confirmed malaria case incidence, and a stronger association where mSpray was used; the use of 3 GIRS insecticides may be enhancing the observed effect, likely due to vector increased susceptibility to the insecticide. The mSpray electronic tool also appeared to improve the effectiveness of IRS. The greater effect observed in IRS areas using mSpray may be due to
Table 4 Random effects Poisson regression results

\begin{tabular}{lllr}
\hline Parameter & $\begin{array}{l}\text { Incidence rate } \\
\text { ratio (IRR) }\end{array}$ & $\begin{array}{l}\text { 95\% Confidence } \\
\text { Interval }\end{array}$ & P-value \\
\hline $\begin{array}{llll}\text { Province } \\
\text { Eastern (ref.) }\end{array}$ & & & \\
Luapula & 1.72 & $1.49-1.98$ & $<0.001$ \\
Muchinga & 1.27 & $1.11-1.45$ & 0.001 \\
Northern & 1.04 & $0.89-1.23$ & 0.621 \\
IRS & & & \\
None (ref.) & & & \\
IRS & 0.91 & $0.84-0.98$ & 0.019 \\
IRS w/ mSpray & 0.75 & $0.66-0.86$ & $<0.001$ \\
Rainfall & 1.00 & $1.00-1.00$ & 0.017 \\
EVI & 1.50 & $0.88-2.56$ & 0.136 \\
Year & & & \\
2014 (ref.) & & & 0.004 \\
2015 & 1.12 & $1.04-1.21$ & 0.001 \\
2016 & 1.16 & $1.06-1.24$ & 0.050 \\
2017 & 1.11 & $1.00-1.19$ & 0.260 \\
Total tested & 1.00 & $0.99-1.00$ & \\
Total confirmed & 0.99 & $0.99-1.00$ & \\
$\quad$ previous year & & & \\
\hline & & & \\
\hline
\end{tabular}

achieving more even, and overall higher, population level household coverage.

There are potential biases in operational coverage reporting when reliant on self-reported programme data. 
For example, spraying of houses requires a high level of training to produce a consistent and appropriate coating of insecticide on walls of varying sizes and materials. When operational definitions are used to define spray coverage it is clear that some houses or structures defined as sprayed will actually be inadequately sprayed with insecticide, perhaps due in part to a failure to spray all of the appropriate rooms or surfaces. In addition, without adequate supervision, non-sprayed structures may be reported as sprayed. Behavioural factors and demand may also contribute to bias in reporting coverage. Individuals may refuse entry to spray teams or be absent at the time of the visit; individuals may also re-paint or re-plaster walls shortly after allowing their homes to be sprayed [22]. Little quantitative information is available on these phenomena, but what is available indicates that large fractions of the population may engage in behaviour that prevents or reduces IRS effect [22].

Evaluation of IRS programmes from observational data has proven challenging because in most cases the programme is highly targeted to higher malaria burden areas, leading to large amounts of endogeneity in observational studies [23]. Though several studies have used alternative approaches to this [24], standard statistical methods are likely to result in counterintuitive estimates of positive associations between malaria prevalence or incidence and IRS application largely because of this kind of targeting, though targeting is highly desirable from a control perspective. Alternative study designs such as interrupted time series, differences-in-differences, and spatio-temporal modelling approaches can, sometimes, mitigate this kind of endogeneity and yield sensible estimates of IRS effects from observational data.

The effects of IRS with Actellic in this setting were much larger than the estimated effect of IRS with other chemicals, especially pyrethroids, observed elsewhere. This may be due to insecticide resistance reducing the susceptibility of the local vectors to IRS chemicals other than Actellic, the extended duration of effectiveness of Actellic on house walls compared to the other IRS chemicals used, or a combination of both these factors. This additional effect comes, however, at an increased cost. Actellic is currently a much higher priced active ingredient than all other WHO-approved IRS active ingredients. The extent to which this extra effectiveness would be considered beneficial relative to other vector control alternatives will require more detailed investigation of the cost and cost-effectiveness of IRS with Actellic as compared improved LLIN coverage, use of larvicides or improving access to prompt and effective care. In this context, this study has estimated an effectiveness of IRS with Actellic that is similar to that found for LLINs under conditions of limited or no insecticide resistance [1], although any variations in insecticide resistance from the previously used insecticide could be important.

While this study suggests a positive impact of 3GIRS on malaria incidence, the results should be interpreted with some caution, as the mSpray intervention was done in one province only, so there may be an underlying provincial effect in place. Secondly, this was an observational study; despite attempts to mitigate the effects of endogeneity due to IRS targeting, and to adjust for all appropriate confounding factors, the estimate of IRS effects found here are the result of associations rather than the results of a controlled experiment. Third, several unnamed 'other' facility catchment areas received IRS but could not be identified through the available data sources. Some of these facilities may have erroneously been included in the comparison group, leading to information bias resulting from misclassification. However, as this would tend to bias results of effect toward null results, these results are likely conservative. Fourth, an independent comparison of population level IRS coverage across areas using different reporting schemes to quantitatively assess whether coverage drove the greater effect observed in mSpray areas was lacking. Lastly, there was limited data on IRS in the period before the intervention activity, so it is not known if these catchment areas reflect true baseline values or not.

\section{Conclusions}

IRS using Actellic-CS, the first approved and widely used 3GIRS chemical, appears to be associated with reduced malaria incidence in Zambia. The use of the mSpray tool appears to improve the effectiveness of the IRS programme, possibly through allowing for better targeting and improved population level coverage. The results of this study lend credence to the anecdotal evidence of the effectiveness of 3GIRS using Actellic, and the importance of exploring new platforms for improving effective population coverage of areas targeted for spraying.

\section{Abbreviations \\ IRS: Indoor residual spraying; IRR: Incidence rate ratio; 3GIRS: Third generation pyrethroid-free insecticides for IRS; LLINs: Long-lasting insecticidal nets; WHO: World Health Organization; GPIRM: Global Plan for Insecticide Resistance Man- agement; NMEC: National Malaria Elimination Centre; ITN: Insecticide-treated bed net; PMI: President's Malaria Initiative; AIRS: PMI Africa Indoor Residual Spraying Project; OPD: Outpatient department; HMIS: Health management information system; EVI: Enhanced vegetation index.}

\section{Acknowledgements}

The authors would like to thank Javan Chanda with MACEPA, Dr Mutinta Mudenda, Dr Anthony Yeta, and Reuben Zulu with the Zambia Ministry of Health National Malaria Elimination Centre, Brian Chirwa with PMI, and Kelvin Mwenya for their support and guidance with this project.

\section{Authors' contributions}

$A B, J Y, T E, B H$ and JM designed the study. JY and $A B$ analysed the data. JK, TE, $J M, J Y, B H, S S$, and $A B$ co-wrote the paper and provided edits and comments 
to the manuscript. All authors participated in writing the manuscript and approved the submitted version. All authors read and approved the final manuscript.

\section{Funding}

Funding for this analysis was supported through grant funding to IVCC, PATH and Tulane University through the NgenIRS project from UNITAID.

\section{Availability of data and materials}

The datasets used and/or analysed during the current study are available from the corresponding author on reasonable request.

\section{Declarations}

\section{Ethics approval and consent to participate}

Not applicable.

\section{Consent for publication}

Not applicable.

\section{Competing interests}

All authors declare that they do not have competing interests.

\section{Author details}

${ }^{1}$ Center for Applied Malaria Research and Evaluation, Department of Tropical Medicine, School of Public Health and Tropical Medicine, Tulane University, 1440 Canal Street, Suite 2320, New Orleans 70112, USA. ${ }^{2}$ PATH Malaria Control and Elimination Partnership in Africa (MACEPA), National Malaria Elimination Centre, Chainama Hospital College Grounds, Lusaka, Zambia. ${ }^{3}$ National Malaria Elimination Centre, Ministry of Health, Lusaka, Zambia. ${ }^{4}$ Global Health Sciences, University of California, San Francisco, CA, USA

Received: 12 June 2020 Accepted: 24 March 2021

Published online: 01 April 2021

\section{References}

1. Lim SS, Fullman N, Stokes A, Ravishankar N, Masiye F, Murray CJL, et al. Net benefits: a multicountry analysis of observational data examining associations between insecticide-treated mosquito nets and health outcomes. PLoS Med. 2011:8:e1001091.

2. Pluess B, Tanser FC, Lengeler C, Sharp BL. Indoor residual spraying for preventing malaria. Cochrane Database Syst Rev. 2010;4:CD006657.

3. Ranson H, N'Guessan R, Lines J, Moiroux N, Nkuni Z, Corbel V. Pyrethroid resistance in African anopheline mosquitoes: what are the implications for malaria control? Trends Parasitol. 2011;27:91-8.

4. WHO. Global Malaria Programme. Global plan for insecticide resistance management in malaria vectors. Geneva: World Health Organization; 2012.

5. Lengeler $C$. Insecticide-treated bed nets and curtains for preventing malaria. Cochrane Database Syst Rev. 2004;2:CD000363.

6. Yukich JO, Lengeler C, Tediosi F, Brown N, Mulligan J-A, Chavasse D, et al. Costs and consequences of large-scale vector control for malaria. Malar J. 2008;7:258.

7. Rehman AM, Coleman M, Schwabe C, Baltazar G, Matias A, Gomes IR, et al. How much does malaria vector control quality matter: the epidemiological impact of holed nets and inadequate indoor residual spraying. PLoS One. 2011;6:e19205.

8. Bridges DJ, Pollard D, Winters AM, Winters B, Sikaala C, Renn S, et al. Accuracy and impact of spatial aids based upon satellite enumeration to improve indoor residual spraying spatial coverage. Malar J. 2018;17:93.

9. Zambia Ministry of Health. National Malaria Elimination Strategic Plan 2017-2021: a strategy to move from accelerated burden reduction to malaria elimination in Zambia. Lusaka; 2017 pp. 1-48.

10. Chanda E, Thomsen EK, Musapa M, Kamuliwo M, Brogdon WG, Norris DE, et al. An operational framework for insecticide resistance management planning. Emerg Infect Dis. 2016;22:773-9.

11. Chanda E, Hemingway J, Kleinschmidt I, Rehman AM, Ramdeen V, Phiri FN. Insecticide resistance and the future of malaria control in Zambia. PLOS ONE. 2011;6:e24336.

12. Abong'o B, Gimnig JE, Torr SJ, Longman B, Omoke D, Muchoki M, et al. Impact of indoor residual spraying with pirimiphos-methyl (Actellic $300 C S$ ) on entomological indicators of transmission and malaria case burden in Migori County, western Kenya. Sci Rep. 2020;10:4518.

13. Rowland M, Boko P, Odjo A, Asidi A, Akogbeto M, N'Guessan R. A new long-lasting indoor residual formulation of the organophosphate insecticide pirimiphos methyl for prolonged control of pyrethroid-resistant mosquitoes: an experimental hut trial in Benin. PLOS ONE. 2013;8:e69516.

14. Mashauri FM, Manjurano A, Kinung'hi S, et al. Indoor residual spraying with micro-encapsulated pirimiphos-methyl (Actellic ${ }^{\circledR}$ 300CS) against malaria vectors in the Lake Victoria basin, Tanzania. PLoS ONE. 2017;12:e0176982.

15. Chanda E, Mzilahowa T, Chipwanya J, Mulenga J, Ali D, Troell P, et al. Preventing malaria transmission by indoor residual spraying in Malawi: grappling with the challenge of uncertain sustainability. Malar J. 2015;14:254.

16. WHO. World Malaria Report 2016. Geneva: World Health Organization; 2016

17. Chizema-Kawesha E, Miller JM, Steketee RW, Mukonka VM, Mukuka C, Mohamed AD, et al. Scaling up malaria control in Zambia: progress and impact 2005-2008. Am J Trop Med Hyg. 2010;83:480-8.

18. PATH, Zambia National Malaria Elimination Centre. Zambia National Malaria Indicator Survey 2015. Lusaka, 2016:1-152.

19. PATH, Zambia National Malaria Elimination Centre. Zambia National Malaria Indicator Survey 2018. Lusaka, 2019:1-144.

20. PMI. Zambia 2016 End of Spray Report. Lusaka, 2017:1-83.

21. PATH, Zambia National Malaria Elimination Centre. Zambia National Malaria Indicator Survey 2012. Lusaka, 2012:1-134.

22. Mnzava AEP, Mnzava AE, Ntuli, Sharp B, Ngxongo S, Mthembu J, et al. House spraying and replastering in KwaZuluNatal. S Afr Med J. 1998:88:1024-8.

23. Bennett A, Yukich J, Miller JM, Keating J, Moonga H, Hamainza B, et al. The relative contribution of climate variability and vector control coverage to changes in malaria parasite prevalence in Zambia 2006-2012. Parasit Vectors. 2016;9:431

24. Over M, Bakote'e B, Velayudhan R, Wilikai P, Graves PM. Impregnated nets or DDT residual spraying? Field effectiveness of malaria prevention techniques in Solomon Islands, 1993-1999. Am J Trop Med Hyg. 2004;71:214-23.

\section{Publisher's note}

Springer Nature remains neutral with regard to jurisdictional claims in published maps and institutional affiliations. 